\title{
La lipocalina asociada con la gelatinasa de neutrófilos como factor temprano de predicción de la función retardada del injerto renal
}

\author{
John Fredy Nieto-Ríos', Lina María Serna-Higuita1', Catalina Ocampo-Kohn¹, Arbey Aristizábal-Alzate', \\ Catalina Vélez-Echeverry ${ }^{1}$, Juan José Vanegas-Ruíz¹, Ana María Bedoya², Sandra Lucía Lopera², \\ Nury Rojano-Held ${ }^{1}$, Nelson Darío Giraldo ${ }^{3}$, Gustavo Adolfo Zuluaga-Valencia ${ }^{1}$ \\ Grupo de Nefrología y Trasplante Renal, Hospital Pablo Tobón Uribe, Universidad de Antioquia, Medellín, Colombia \\ 2 Laboratorio Clínico, Hospital Pablo Tobón Uribe, Medellín, Colombia \\ 3 Cuidado Crítico, Hospital Pablo Tobón Uribe, Medellín, Colombia
}

Introducción. La función retardada del injerto renal se presenta en 20 a 50 \% de los trasplantes renales. Objetivo. Describir el comportamiento de la lipocalina urinaria asociada a la gelatinasa de neutrófilos en receptores de trasplante renal de donante fallecido y compararlo con el porcentaje de descenso de la creatinina sérica para detectar tempranamente la función retardada del injerto renal.

Materiales y métodos. Se evaluaron los niveles de lipocalina urinaria asociada a la gelatinasa de neutrófilos en una cohorte prospectiva 1, 12, 24 y 48 horas después del trasplante renal, y se compararon diariamente con los de la creatinina sérica hasta el quinto día del trasplante.

Resultados. Se incluyeron en el estudio 79 pacientes con trasplante renal de donante fallecido. La función retardada del injerto renal se presentó en 13 pacientes (16,5\%) y cinco de ellos $(6,3 \%)$ necesitaron diálisis en la primera semana. Los valores de lipocalina urinaria asociada a la gelatinasa de neutrófilos en todos los puntos de corte fueron mayores en los pacientes con función retardada del injerto renal ( $p=0,526, p=0,049, p=0,032$ y $p=0,001$, respectivamente). Se estableció un valor de más de $120 \mathrm{ng} / \mathrm{ml}$ a las 48 horas como factor de predicción de la función retardada del injerto, con una sensibilidad de $75 \%$ y una especificidad de $71 \%$; el valor de creatinina sérica que mejor discriminó la función retardada se presentó a las 48 horas (59,5\%), con una sensibilidad de $92 \%$ y una especificidad de $83 \%$. En la regresión logística los únicos valores significativos para predecir la función retardada del injerto renal fueron los de la creatinina serica.

Conclusión. Los niveles de lipocalina urinaria asociada a la gelatinasa de neutrófilos a las 48 horas del trasplante renal predijeron la función retardada, incluida la necesidad de diálisis, pero no fueron superiores a los de la creatinina sérica para la detección temprana.

Palabras clave: trasplante de riñón, funcionamiento retardado del injerto, creatinina, lesión renal aguda, donantes de tejidos.

doi: http://dx.doi.org/10.7705/biomedica.v36i2.2703

\section{Neutrophil gelatinase-associated lipocalin as an early predictor of delayed graft function}

Introduction: Delayed graft function occurs in about 20 to 50 percent of kidney transplants.

Objective: To describe the behavior of urinary neutrophil gelatinase-associated lipocalin (NGALu) in deceased-donor renal transplant recipients and to compare this indicator with the percentage of creatinine decrease $(\mathrm{PdC})$ for the early detection of delayed graft function.

Materials and methods: NGALu levels were evaluated in a prospective cohort in the first, 12th, 24th and 48th hours after kidney transplant, and compared with the daily PdC until day 5.

Results: We included 79 patients in the study. Delayed graft function occurred in 13 patients (16.5\%), and five patients $(6.3 \%)$ required dialysis in the first week. NGALu levels at all cut-off points were higher in patients with delayed graft function ( $p=0.526, p=0.049, p=0.032$, and $p=0.001$ ). NGALu levels above $120 \mathrm{ng} / \mathrm{ml}$ at 48 hours predicted delayed graft function with a sensitivity of $75 \%$ and a

\footnotetext{
Contribución de los autores:

John Fredy Nieto-Ríos y Lina María Serna-Higuita: concepción y diseño del estudio, análisis e interpretación de los datos, redacción y revisión del artículo

John Fredy Nieto-Ríos, Lina María Serna-Higuita, Catalina Ocampo-Kohn, Arbey Aristizábal-Alzate, Catalina Vélez-Echeverry, Juan José Vanegas-Ruíz, Nury Rojano-Held, Gustavo Adolfo Zuluaga-Valencia: reclutamiento y seguimiento de los pacientes Ana María Bedoya y Sandra Lucía Lopera: procesamiento de las muestras de laboratorio Lina María Serna-Higuita, Nury Rojando-Held y Nelson Darío Giraldo: análisis estadístico de los datos Todos los autores aprobaron la versión final del manuscrito.
} 
specificity of $71 \%$. A PdC of $59.5 \%$ best discriminated the delayed graft function, with a sensitivity of $92 \%$ and a specificity of $83 \%$ at 48 hours. Using logistic regression for the adjusted delayed graft function, the only significant values to predict it were those of PdC.

Conclusions: NGALu levels measured at 48 hours after renal transplantation predicted delayed graft function, including the need for dialysis; however, this marker was not superior to the PdC for early detection.

Key words: Kidney transplant, delayed graft function, creatinine, acute kidney injury, tissue donors. doi: http://dx.doi.org/10.7705/biomedica.v36i2.2703

La función retardada del injerto renal (1-4) es una complicación que se presenta en 20 a $50 \%$ de los trasplantes de riñón de donante fallecido $(5,6)$. Se le considera una lesión por isquemia y reperfusión (5) cuyos mecanismos fisiopatológicos involucran el estrés oxidativo, la disfunción mitocondrial y la activación inmunológica (7), lo que no permite la recuperación completa de las células renales, predispone a la atrofia y la muerte celular (8), aumenta el riesgo de rechazo agudo y crónico (3) y la estancia hospitalaria, y reduce la supervivencia del injerto renal (3,6,9-12).

Aunque en la actualidad se sabe mucho sobre la fisiopatología de la función retardada del injerto, son pocas las estrategias de tratamiento conocidas (6); algunos tratamientos exitosos de la lesión por reperfusión en modelos animales no han sido efectivos en humanos $(5,12)$, lo cual se explicaría por la detección tardía de la función retardada del injerto (8), ya que los biomarcadores actuales, que incluyen la creatinina sérica y la diuresis, son poco sensibles para predecirla $(11,13)$. El hallazgo de nuevos biomarcadores que detecten en forma no invasiva y temprana la función retardada del injerto, permitiría la implementación de tratamientos para limitar el daño $(3,6,14,15)$ y mejorar el resultado a largo plazo del injerto renal $(8,16)$.

Uno de los biomarcadores más opcionados es la lipocalina urinaria asociada a la gelatinasa de neutrófilos (LAGN urinaria) $(17,18)$, la cual es producida principalmente por los neutrófilos y las células del epitelio tubular renal (18). En algunos estudios en pacientes con trasplante de riñón se ha sugerido que la LAGN urinaria podría ser superior a la creatinina para la detección temprana de la función retardada del injerto, ya que su elevación puede registrarse dos horas después del trasplante renal $(3,5,6,8,14,19,20)$.

\section{Correspondencia:}

Lina María Serna, Calle 78b № 69-240, Medellín, Colombia Teléfono: (574) 4459902

Im.serna@hotmail.com

Recibido: 06/02/15; aceptado: 09/09/15
En el Hospital Pablo Tobón Uribe predominan los trasplantes de donante fallecido, grupo en el que la frecuencia de la función retardada del injerto es mayor $(5,6)$. Por este motivo se necesitan marcadores que permitan su detección temprana. Este estudio tuvo como objetivo determinar si los valores de la LAGN urinaria predecían la función retardada del injerto más tempranamente que el porcentaje de descenso de la creatinina sérica.

\section{Materiales y métodos}

Se hizo un estudio prospectivo de cohorte en el Hospital Pablo Tobón Uribe, institución de alto nivel de complejidad en Medellín, Colombia; se incluyeron pacientes mayores de 12 años que habían recibido un trasplante renal de donante fallecido entre noviembre de 2012 y diciembre de 2013. Se excluyeron los pacientes que presentaron anuria durante las primeras seis horas después del trasplante.

Se recopilaron los datos de edad, sexo, causa de la enfermedad renal terminal, número de incompatibilidades del sistema de antígenos leucocitarios humanos (Human Leukocyte Antigen, HLA), tiempo de isquemia fría y caliente, transfusiones y trasplantes previos, uso de terapia dialítica previa al trasplante, inmunosupresión utilizada (inducción y mantenimiento) y calidad del donante (si cumplían los criterios extendidos o no lo hacían).

Se recolectaron muestras de orina para la medición de la LAGN urinaria 1, 12, 24 y 48 horas después del trasplante. Estas muestras se procesaron en el laboratorio del hospital de la siguiente forma: se centrifugaron a 400 revoluciones por minutos durante cinco minutos y se almacenaron a menos de $70^{\circ} \mathrm{C}$ durante un máximo de tres meses hasta su procesamiento. La determinación de la LAGN urinaria se hizo mediante inmunoensayo turbidimétrico (Sentinel Diagnostics, REF 1P9300) sobre la plataforma Architect $18000^{\circledR}$ de Abbott. Los niveles de creatinina sérica se registraron diariamente antes y después del trasplante.

El resultado evaluado fue la presencia o ausencia de la función retardada de injerto, definida como una disminución de la creatinina sérica menor de $10 \%$ 
durante tres días consecutivos (1), la necesidad de diálisis en la primera semana del trasplante renal $(1,3)$ o un nivel de creatinina sérica mayor de $3 \mathrm{mg} /$ dl al quinto día del trasplante (1).

Se describieron las características demográficas según la presencia o ausencia de la función retardada del injerto. Se hizo una descripción estadística utilizando medias y desviación estándar o medianas y rangos intercuartílicos, de acuerdo con la distribución de los datos evaluados mediante la prueba de Shapiro Wills; las variables continuas se analizaron con base en la diferencia de medias o la prueba $U$ de Mann-Withney y las variables categóricas se compararon con la prueba de ji al cuadrado o la de Fisher. Los resultados se consideraron estadísticamente significativos cuando arrojaron un valor de $p<0,05$.

Se evaluaron los valores de la LAGN urinaria agrupados según la presencia o ausencia de la función retardada del injerto y, por último, se determinó el mejor punto de corte y el tiempo óptimo para ambos marcadores. Para el diagnóstico de la función retardada del injerto, se evaluó la curva de la característica operativa del receptor (Receiving Operating Characteristic, ROC) de la LAGN urinaria y se comparó con el porcentaje de descenso de la creatinina sérica, el cual se estableció tomando como valor inicial el registrado en el momento del trasplante.

Se utilizó un modelo de regresión logística en el que la variable dependiente fue la presencia de la función retardada del injerto, y las variables independientes fueron los valores de la LAGN urinaria y el porcentaje de descenso de la creatinina sérica. Se ajustaron algunas variables de confusión encontradas en la literatura científica y se ingresaron al modelo las variables con $p<0,25$ en el análisis bivariado. Se verificaron los supuestos de homocedasticidad, normalidad e independencia de los residuales y se utilizó la prueba de Wald para evaluar la significación estadística del modelo de regresión logística. Para el análisis estadístico se utilizó el programa Stata ${ }^{\circledR}$.

\section{Consideraciones éticas}

El estudio fue aprobado por el Comité de Ética en Investigaciones del Hospital Pablo Tobón Uribe.

\section{Resultados}

Entre noviembre de 2012 y diciembre de 2013 se hicieron 83 trasplantes renales en el Hospital Pablo Tobón Uribe. Dos pacientes fueron excluidos por anuria en las primeras horas y otros dos por ser menores de 12 años, es decir, quedaron 79 pacientes para el análisis. Todos los trasplantes fueron de donante fallecido, pero ninguno de criterio extendido; 44 pacientes eran hombres $(55,7 \%)$, la media de la edad fue de 39,6 años con una desviación estándar (DE) de 14,18; se trataba del primer trasplante en $91,1 \%$ de los casos y del segundo en $8,9 \% ; 82,3 \%$ de los pacientes estaba en diálisis antes del trasplante, con una mediana de 23,5 meses (25-75:4-48); 51,9\% había recibido transfusiones previas y la media del tiempo de isquemia fría fue de 15,04 horas (DE $\pm 5,84)$.

La función retardada del injerto se presentó en 13 pacientes (16,5\%) y cinco de ellos $(6,3 \%)$ necesitaron diálisis en la primera semana. Las características demográficas agrupadas con base en la presencia o la ausencia de dicha función, se muestran en el cuadro 1.

Al evaluar los valores de la LAGN urinaria agrupados según la presencia o la ausencia de la función retardada del injerto, se encontró que en todos los puntos de corte dichos valores fueron mayores en los pacientes con función retardada, y alcanzaron la significación estadística a las 24 y 48 horas del trasplante renal $(p=0,031$ y $p=0,001$, respectivamente) (figura 1 ).

Según la prueba de diferencias de las medias, los valores de la LAGN urinaria agrupados con base en la presencia o la ausencia de la función retardada del injerto renal fueron los siguientes: a la hora del trasplante, $p=0,526$; a las 12 horas, $p=0,049$; a las 24 horas, $p=0,032$, y a las 48 horas, $p=0,001$.

Se buscó establecer el mejor punto de corte para predecir la función retardada del injerto, y se encontró que un valor de LAGN de más de $120 \mathrm{ng} /$ $\mathrm{ml}$ a las 48 horas la predecía con una sensibilidad de $75 \%$ y una especificidad de $70 \%$ (área bajo la curva de 0,798). El porcentaje de descenso de la creatinina sérica que mejor discriminó la función retardada del injerto fue de $59,5 \%$ a las 48 horas, con una sensibilidad de $92 \%$ y una especificidad de $83 \%$ (área bajo la curva de 0,925). Al comparar las curvas ROC de la LAGN urinaria y el porcentaje de descenso de la creatinina sérica, este último fue un mejor marcador (figura 2).

Los puntos de corte escogidos para categorizar la LAGN urinaria y el porcentaje de descenso de la creatinina sérica a las 48 horas del trasplante renal, fueron de $120 \mathrm{ng} / \mathrm{ml}$ y $59,5 \%$, respectivamente. Al evaluar la razón de probabilidades (odds ratio, 
Cuadro 1. Análisis bivariado agrupado según la presencia o ausencia de la función retardada del injerto renal

\begin{tabular}{|c|c|c|c|}
\hline Variable & FRI $(n=13)$ & FAI $(n=66)$ & $\mathbf{p}^{\star *}$ \\
\hline Sexo (número y porcentaje de hombres) & $9 \quad(69,2)$ & $35 \quad(53)$ & $0,28(a)$ \\
\hline Edad en años en el momento del trasplante, media (DE) & $38,6( \pm 13,8)$ & $39,7( \pm 14,3)$ & $0,78(d)$ \\
\hline Antecedentes de transfusiones previas, $\mathrm{n}(\%)$ & $6(46,1)$ & $34 \quad(51,5)$ & $0,88(a)$ \\
\hline Segundo trasplante, $\mathrm{n}(\%)$ & $2(15,4)$ & $5(7,6)$ & $0,32(b)$ \\
\hline Diálisis previa, $\mathrm{n}(\%)^{*}$ & $12(92,3)$ & $53(80,3)$ & $0,44(b)$ \\
\hline Edad en años del donante, mediana (RIQ) & $27 \quad(24-42)$ & $26 \quad(19-41)$ & $0,51(c)$ \\
\hline Tres o menos incompatibilidades en el HLA, $n$ (\%) & $1(6,67)$ & $14 \quad(93,3)$ & $0,27(b)$ \\
\hline Cuatro o más incompatibilidades en el HLA, n (\%) & $11 \quad(17,5)$ & $52(82,5)$ & $0,27(b)$ \\
\hline Creatinina del donante, mediana (RIQ) & $0,8(0,68-0,96)$ & $0,8(0,6-1,1)$ & $0,70(\mathrm{c})$ \\
\hline Tiempo de isquemia fría en horas, media (DE) & $18,2(6,7)$ & $14,4(5,49)$ & $0,03(d)$ \\
\hline Tiempo en minutos de isquemia caliente, mediana (RIQ) & $30 \quad(30-28,75)$ & $30 \quad(30-36,2)$ & 0,97 (c) \\
\hline Inducción con basiliximab, n (\%) & $4 \quad(30,8)$ & $21 \quad(31,8)$ & $0,61(b)$ \\
\hline Inducción con timoglobulina, n (\%) & $9 \quad(69,2)$ & $45 \quad(68,2)$ & $0,61(b)$ \\
\hline
\end{tabular}

FRI: función retardada del injerto renal; FAI: función adecuada del injerto renal; DE: desviación estándar; RIQ: rango intercuartílico; HLA: complejo mayor de histocompatibilidad. * Antecedentes de terapia de reemplazo renal antes del trasplante. ${ }^{\star}{ }^{\star} E \mathrm{l}$ análisis bivariado se hizo con las siguientes pruebas estadísticas: (a) ji al cuadrado, (b) test de Fisher, (c) prueba U de Mann-Whitney, (d) t de Student.

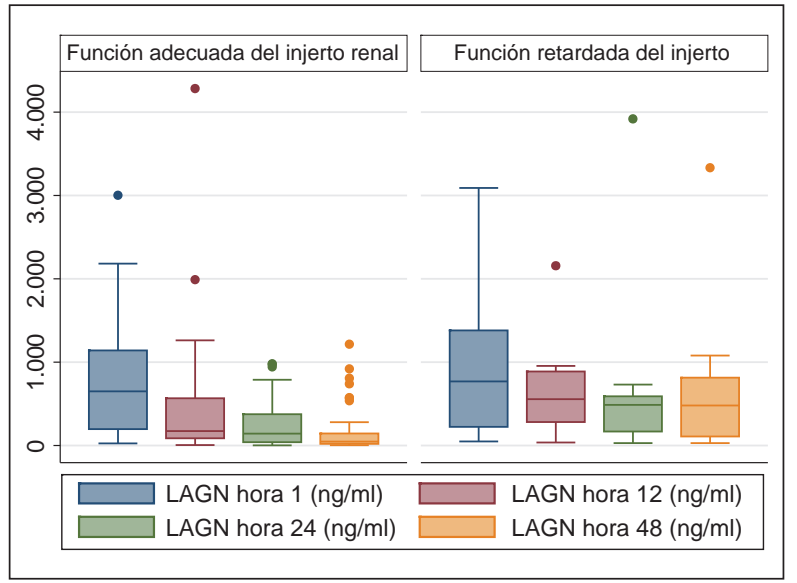

Figura 1. Valores de lipocalina urinaria asociada a la gelatinasa de neutrófilos después del trasplante renal, agrupados según la presencia o ausencia de la función retardada del injerto renal

OR) de ambos puntos de corte, la correspondiente a tener un valor positivo de LAGN urinaria para la función retardada del injerto fue de $5,25\left(\mathrm{IC}_{95 \%}\right.$ $1,55-17,81)$ y la de tener un porcentaje de descenso de la creatinina sérica positivo fue de $27,5\left(\mathrm{IC}_{95 \%}\right.$ 3,79-199,75).

Se hizo un análisis de regresión logística exploratorio ajustado según el número de trasplantes y el tiempo de isquemia fría, en el cual el porcentaje de descenso de la creatinina sérica fue el único valor significativo para predecir la función retardada del injerto (cuadro 2).

\section{Discusión}

En este estudio se evaluaron los valores de la LAGN urinaria de una cohorte prospectiva de pacientes con trasplante renal de donantes fallecidos que no

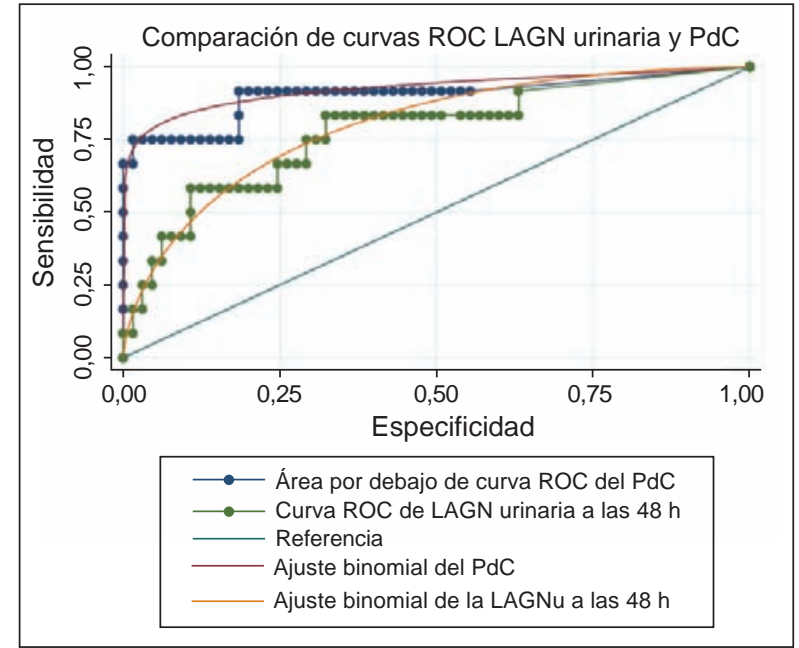

Figura 2. Curvas ROC de la comparación entre la lipocalina urinaria asociada a la gelatinasa de neutrófilos y el porcentaje de depuración de la creatinina sérica

PdC: porcentaje de depuración de la creatinina sérica; LAGNu: lipocalina urinaria asociada a la gelatinasa de neutrófilos

respondían al criterio extendido. Se encontró que el valor de la LAGN urinaria medido a las 48 horas del trasplante predecía la función retardada del injerto; sin embargo, al compararlo con el porcentaje de descenso de la creatinina sérica, este detectó con mayor exactitud el riesgo de dicho retardo, hallazgo similar al de estudios como el de Kaufeld, et al., quienes evaluaron 105 pacientes con trasplante renal cuyos niveles de LAGN urinaria fueron dos veces más altos en aquellos con lesión renal que en los que no la presentaron. En este estudio, sin embargo, muchos valores se cruzaron entre los dos grupos y a los seis meses no se encontraron diferencias $(21,22)$. 
Cuadro 2. Regresión logística para detectar la presencia de la función retardada del injerto renal

\begin{tabular}{lcccc}
\hline Variable FRI & OR & $\begin{array}{c}\text { Error } \\
\text { estándar }\end{array}$ & $\mathbf{p}$ & $\mathbf{I C}_{95 \%}$ \\
\hline LAGNu & 0,622 & 0,589 & 0,616 & $0,111-0,700$ \\
PdC & 0,016 & 0,02 & 0,001 & $0,001-0,218$ \\
$\begin{array}{l}\text { Número de } \\
\text { trasplantes }\end{array}$ & 1,157 & 0,88 & 0,054 & $0,997-1,343$ \\
$\begin{array}{l}\text { Tiempo de } \\
\text { isquemia fría }\end{array}$ & 0,437 & 0,51 & 0,483 & $0,043-4,406$ \\
Constante & 0,251 & 0,46 & 0,45 & $0,007-9,115$ \\
\hline
\end{tabular}

Logaritmo de verosimilitud=-20,2234; pseudo $R^{2}=0,39$

FRI: función retardada del injerto renal; OR: razón de momios; LAGNu: lipocalina urinaria asociada a la gelatinasa de neutrófilos; PdC: porcentaje de depuración de la creatinina sérica

La LAGN es una proteína de $25 \mathrm{kDa}$ (23) conformada por ocho cadenas beta con capacidad de transportar pequeñas moléculas. La LAGN es producida principalmente por los neutrófilos y por las células del epitelio tubular renal (18). En estudios recientes se han encontrado diferentes clases de esta proteína: una forma monomérica de $25 \mathrm{kDa}$, un homodímero de $45 \mathrm{kDa}$ y un heterodímero de $135 \mathrm{kDa}$; los neutrófilos producen el homodímero y las células tubulares renales producen los heterodímeros y monómeros $(23,24)$. Los niveles de LAGN urinaria están compuestos por la proteína secretada por las células tubulares renales, pero también, por la producida por los neutrófilos filtrados al riñón (24). La función retardada del injerto es una lesión por 'injuria' y reperfusión, y por este motivo, la LAGN se ha postulado como una herramienta no invasiva para su detección, pues su relación con la función retardada del injerto puede registrarse incluso una hora después de la reperfusión $(2,6,10,25,26)$, en tanto que la creatinina se demora entre uno y tres días en alterarse (2).

Entre las funciones de la LAGN se han encontrado el transporte de hierro (5), la regulación del crecimiento y la diferenciación celular, pues en su calidad de componente de la inmunidad innata, inhibe el crecimiento bacteriano y transporta el hierro dentro de las células durante los procesos infecciosos (17). Se cree que la LAGN induce inhibición del crecimiento bacteriano, disminución de la apoptosis y aumento de la proliferación en los túbulos renales, lo cual constituye una posible vía de protección renal (23). En los estudios en ratones con isquemia e 'injuria' por reperfusión, se encontró que al administrar LAGN recombinante por vía intraperitoneal, el daño de los tejidos renales y el número de células apoptóticas eran menores, que había un incremento en la proliferación de las células tubulares (19) y que, además, el valor de la creatinina sérica era más bajo (19), hallazgos que sugieren que la LAGN aumenta como reacción a la 'injuria' y puede desempeñar un papel importante en la recuperación renal $(5,19)$. En el 2009 se publicó un metanálisis de 19 estudios con un total de 2.538 participantes, en el cual se evaluaron los valores de la LAGN en orina y en sangre de pacientes con 'injuria' renal aguda de diferentes causas (15); los autores encontraron que la detección de niveles de LAGN sérica y urinaria de $150 \mathrm{ng} / \mathrm{ml}$ era una herramienta útil para predecir la necesidad de recurrir a la terapia de reemplazo renal, con una razón de momios (OR) de 12,9 $(15,27)$.

Los hallazgos de este estudio son similares a los reportados en varios otros en los que se ha encontrado que los valores de LAGN urinaria son predictores de la función retardada del injerto renal $(10,28)$, pero en este estudio no fueron mejores que el porcentaje de descenso de la creatinina sérica. En un estudio de 27 pacientes con trasplante renal de donante vivo relacionado, Rahimzadeh, et al., encontraron que un valor de LAGN urinaria de $174 \mathrm{ng} / \mathrm{ml}$ predecía una recuperación lenta de la función renal $(5,10)$. En otro estudio de 64 personas con trasplante renal, Rotzami, et al., encontraron que un valor de LAGN urinaria de $204 \mathrm{ng} / \mathrm{ml}$ dos horas después del trasplante tenía una sensibilidad de $72 \%$ y una especificidad de $67 \%$ para predecir la aparición de 'injuria' renal aguda, en tanto que la creatinina tuvo una mejor sensibilidad y especificidad: $1,55 \mathrm{mg} / \mathrm{dl}$ en el quinto día (2). Hollmen, et al., reportaron que los valores de la LAGN urinaria a las 24 horas del trasplante predecían la función retardada del injerto renal a las tres semanas del trasplante (6). En un estudio de 53 pacientes, Parik, et al., encontraron que un valor de LAGN urinaria de $100 \mathrm{ng} / \mathrm{ml}$ se asociaba a un riesgo de $20 \%$ de función retardada del injerto renal (12). Lee, et al., reportaron que los niveles de la LAGN urinaria en el primer día se asociaron a la función retardada del injerto renal, con una OR de 1,02 y $p=0,0048$ (29). La LAGN urinaria presentaba un área bajo la curva de 0,86 , con un $\mathrm{IC}_{95 \%}$ de $0,75-0,98$, en tanto que el área bajo la curva de la creatinina sérica fue de $0,65\left(\mathrm{IC}_{95 \%}, 0,46-0,83\right)$, con una $p$ de 0,0213 (29). Hall, et al., hicieron un análisis de regresión logística para buscar factores predictores de la función retardada del injerto renal y encontraron una OR ajustada $\left(\mathrm{IC}_{95 \%}\right)$ de 5,1 para la LAGN urinaria (5). 
Entre las limitaciones del estudio deben mencionarse el tamaño de la muestra y el hecho de haberlo llevado a cabo en pacientes con trasplante de donantes fallecidos que cumplían plenamente con los criterios óptimos, en quienes la incidencia de la función retardada del injerto es menor; sin embargo, aunque los valores de la LAGN urinaria fueron mayores cuando se presentó dicho retardo, no fueron superiores que el porcentaje de descenso de la creatinina sérica.

Como conclusión, la medición de la LAGN urinaria a las 24 y 48 horas del trasplante renal en receptores de donantes fallecidos que cumplían con los criterios óptimos, predijo la función retardada del injerto renal, pero no fue superior al porcentaje de descenso de la creatinina sérica para su detección temprana.

\section{Agradecimientos}

A los laboratorios Abbot y Novartis por la donación de los kits de medición de la LAGN urinaria para el estudio.

\section{Conflicto de intereses}

Los autores declaran que no hay conflictos de intereses en este estudio. Los laboratorios Abbot y Novartis no intervinieron en la recolección, el procesamiento ni el análisis de la información.

\section{Financiación}

Hospital Pablo Tobón Uribe y laboratorios Abbot y Novartis.

\section{Referencias}

1. Cohen DJ, Vella JV. Transplantation. NephSAP. 2013;12: 309-99.

2. Rostami Z, Nikpoor M, Einollahi B. Urinary neutrophil gelatinase associated lipocalin (NGAL) for early diagnosis of acute kidney injury in renal transplant recipients. Nephrourol Mon. 2013;5:745-52. http://dx.doi.org/10.5812/ numonthly.9385

3. Siedlecki A, Irish W, Brennan DC. Delayed graft function in the kidney transplant. Am J Transplant. 2011;11:2279-96. http://dx.doi.org/10.1111/j.1600-6143.2011.03754.x

4. Cohen DJ, Vella JoV. NephSAP (Nephrology SelfAssessment Program). Transplantation. 2011;10:531-61.

5. Hall IE, Yarlagadda SG, Coca SG, Wang Z, Doshi M, Devarajan P, et al. IL-18 and urinary NGAL predict dialysis and graft recovery after kidney transplantation. J Am Soc Nephrol. 2010;21:189-97. http://dx.doi.org/10.1681/ASN. 2009030264

6. Hollmen ME, Kyllönen LE, Inkinen KA, Lalla ML, Salmela KT. Urine neutrophil gelatinase-associated lipocalin is a marker of graft recovery after kidney transplantation. Kidney Int. 2011;79:89-98. http://dx.doi.org/10.1038/ki.2010.351
7. Lin M, Li L, Li L, Pokhrel G, Qi G, Rong R, et al. The protective effect of baicalin against renal ischemiareperfusion injury through inhibition of inflammation and apoptosis. BMC Complement Altern Med. 2014;14:19. http://dx.doi.org/10.1186/1472-6882-14-19

8. Choi HM, Park KT, Lee JW, Cho E, Jo SK, Cho WY, et al. Urine neutrophil gelatinase-associated lipocalin predicts graft outcome up to 1 year after kidney transplantation. Transplant Proc. 2013;45:122-8. http://dx.doi.org/10.1016/j. transproceed.2012.05.080

9 Yarlagadda SG, Coca SG, Formica RN, Poggio ED, Parikh CR. Association between delayed graft function and allograft and patient survival: A systematic review and metaanalysis. Nephrol Dial Transplant. 2009;24:1039-47. http:// dx.doi.org/10.1093/ndt/gfn667

10. Rahimzadeh N, Otukesh H, Hoseini R, Sorkhi H, Otukesh $\mathbf{M}$, Hoseini $\mathbf{S}$, et al. Are serum and urine neutrophil gelatinase-associated lipocalin predictive of renal graft function in short term? Pediatr Transplant. 2012;16:796802. http://dx.doi.org/10.1111/j.1399-3046.2012.01770.x

11. Yarlagadda SG, Coca SG, Garg AX, Doshi M, Poggio $\mathrm{E}$, Marcus RJ, et al. Marked variation in the definition and diagnosis of delayed graft function: A systematic review. Nephrol Dial Transplant. 2008;23:2995-3003. http://dx.doi. org/10.1093/ndt/gfn158

12. Parikh CR, Jani A, Mishra J, Ma Q, Kelly C, Barasch J, et al. Urine NGAL and IL-18 are predictive biomarkers for delayed graft function following kidney transplantation. Am J Transplant. 2006;6:1639-45. http://dx.doi.org/10.1111/j. 1600-6143.2006.01352.x

13. Devarajan P. NGAL in acute kidney injury: From serendipity to utility. Am J Kidney Dis. 2008;52:395-9. http://dx.doi. org/10.1053/j.ajkd.2008.07.008

14. Bataille A, Abbas S, Semoun O, Bonnet F, Resche-rigon M, Abboud I, et al. Plasma neutrophil gelatinase-associated lipocalin in kidney transplantation and early renal function. Transplantation. 2011;92:1024-30. http://dx.doi.org/10.1097/ TP.0b013e318230c079

15. Haase M, Bellomo R, Devarajan P, Schlattmann P, HaaseFielitz A. Accuracy of neutrophil gelatinase-associated lipocalin (NGAL) in diagnosis and prognosis in acute kidney injury: A systematic review and meta-analysis. Am J Kidney Dis. 2009;54:1012-24. http://dx.doi.org/10.1053/j. ajkd.2009.07.020

16. Kim SC, Page EK, Knechtle SJ. Urine proteomics in kidney transplantation. Transplant Rev (Orlando). 2014;28:15-20. http://dx.doi.org/10.1016/j.trre.2013.10.004

17. Heyne N, Kemmner S, Schneider C, Nadalin S, Königsrainer A, Häring HU. Urinary neutrophil gelatinaseassociated lipocalin accurately detects acute allograft rejection among other causes of acute kidney injury in renal allograft recipients. Transplantation. 2012;93:1252-7. http:// dx.doi.org/10.1097/TP.0b013e31824fd892

18. Helmersson-Karlqvist J, Ärnlöv J, Larsson A. Day-today variation of urinary NGAL and rational for creatinine correction. Clin Biochem. 2013;46:70-2. http://dx.doi.org/10. 1016/j.clinbiochem.2012.09.022

19. Bolignano D, Donato V, Coppolino G, Campo S, Buemi A, Lacquaniti A, et al. Neutrophil gelatinase-associated lipocalin (NGAL) as a marker of kidney damage. Am J Kidney Dis. 2008;52:595-605. http://dx.doi.org/10.1053/j. ajkd.2008.01.020 
20. Fonseca I, Oliveira JC, Almeida M, Cruz M, Malho A, Martins LS, et al. Neutrophil gelatinase-associated lipocalin in kidney transplantation is an early marker of graft dysfunction and is associated with one-year renal function. J Transplant. 2013;2013:650123. http://dx.doi.org/10. $1155 / 2013 / 650123$

21. Kaufeld JK, Gwinner W, Scheffner I, Haller HG, Schiffer M. Urinary NGAL ratio is not a sensitive biomarker for monitoring acute tubular injury in kidney transplant patients: NGAL and ATI in renal transplant patients. J Transplant. 2012;2012:563404. http://dx.doi.org/10.1155/2012/563404.

22. Vanmassenhove J, Vanholder R, Nagler E, Biesen W Van. Urinary and serum biomarkers for the diagnosis of acute kidney injury: An in-depth review of the literature. Nephrol Dial Transpl. 2013;28:254-73. http://dx.doi.org/10.1093/ndt/ gfs380

23. Singer E, Marko L, Paragas N, Barasch J, Dragun D, Muller N, et al. Neutrophil gelatinase-associated lipocalin: Pathophysiology and clinical applications. Acta Physiol (Oxf). 2013;207:663-72. http://dx.doi.org/10.1111/apha.12054

24. Glassford NJ, Schneider AG, Xu S, Eastwood GM, Young $\mathbf{H}$, Peck $L$, et al. The nature and discriminatory value of urinary neutrophil gelatinase-associated lipocalin in critically ill patients at risk of acute kidney injury. Intensive Care Med. 2013;39:1714-24. http://dx.doi.org/10.1007/ s00134-013-3040-7
25. Adiyanti SS, Loho T. Acute Kidney Injury (AKI) biomarker. Acta Med Indones. 2012;44:246-55.

26. Mishra J, Ma Q, Prada A, Mitsnefes M, Zahedi K, Yang $\mathbf{J}$, et al. Identification of neutrophil gelatinase-associated lipocalin as a novel early urinary biomarker for ischemic renal injury. J Am Soc Nephrol. 2003;14:2534-43. http:// dx.doi.org/10.1097/01.ASN.0000088027.54400.C6

27. Magnusson NE, Hornum M, Jørgensen KA, Hansen JM, Bistrup C, Feldt-Rasmussen B, et al. Plasma neutrophil gelatinase associated lipocalin (NGAL) is associated with kidney function in uraemic patients before and after kidney transplantation. BMC Nephrol. 2012;13:1-8. http://dx.doi. org/10.1186/1471-2369-13-8.

28. Mishra J, Ma Q, Kelly C, Mitsnefes M, Mori K, Barasch $\mathrm{J}$, et al. Kidney NGAL is a novel early marker of acute injury following transplantation. Pediatr Nephrol. 2006;21:856-63. http://dx.doi.org/10.1007/s00467-006-0055-0

29. Lee EY, Kim MS, Park Y, Kim HS. Serum neutrophil gelatinase-associated lipocalin and interleukin-18 as predictive biomarkers for delayed graft function after kidney transplantation. J Clin Lab Anal. 2012;26:295-301. http:// dx.doi.org/10.1002/jcla.21520 